\title{
Antioxidant Properties of Honey and Its Role in Preventing Health Disorder
}

\author{
M.I. Khalil*, S.A. Sulaiman and L. Boukraa \\ Department of Pharmacology, School of Medical Sciences, Universiti Sains Malaysia, Health Campus, 16150 Kubang \\ Kerian, Kelantan, Malaysia
}

\begin{abstract}
Honey is being used since long time both in medical and domestic needs, but only recently its antioxidant property has come to limelight. With increasing demand for antioxidant supply in the food, honey is becoming popular as a source of antioxidant since it is rich in phenolic acids and flavonoids and other antioxidants including glucose oxidase, catalase, ascorbic acid, carotenoid derivatives, organic acids, amino acids and proteins. The antioxidants have several preventative effects against different diseases like cancer, cardiovascular diseases, inflammatory disorders, neurological degeneration, wound healing, infectious diseases and aging, which led to search for foods rich in antioxidants. Various studies on antioxidant properties of honey have been done. The present article is a short review on the antioxidant properties of honey and its role against health disorder.
\end{abstract}

Keywords: Honey, Antioxidant, Phytochemicals, Health disorder.

\section{INTRODUCTION}

Mom always said, "Eat your fruits and vegetables, they'll help you grow big and strong." Although dietary recommendations have changed over the years, this is one bit of advice even Father Time can't ignore.

According to recent studies, antioxidant substances available in various natural sources and foods may actually represent a modern-day "fountain of youth." Evidence suggests that vitamins $\mathrm{C}$ and $\mathrm{E}$, and beta-carotene, a precursor to vitamin A, may reduce the risk of some forms of cancer, heart disease, strokes, and cataracts and may slow the aging process [1].

Honey is a remarkably complex natural liquid that is reported to contain at least 181 substances [2]. The composition of honey is rather variable and primarily depends on the floral source; however, certain external factors also play a role, such as seasonal and environmental factors and processing. Honey is a supersaturated solution of sugars, of which fructose $(38 \%)$ and glucose $(31 \%)$ are the main contributors. A wide range of minor constituents is also present in honey, many of which are known to have antioxidant properties. These include phenolic acids and flavonoids [3, 4], certain enzymes (glucose oxidase, catalase) [2], ascorbic acid [2], carotenoid-like substances [5], organic acids [6], Maillard reaction products [2], and amino acids and proteins [7]. The antioxidant activity of phenolic compounds might significantly contribute to the human health benefits of plant foods $[8,9]$ and beverages such as red wine and tea [9-11].

*Address correspondence to this author at the Department of Pharmacology, School of Medical Sciences, Universiti Sains Malaysia, Health Campus, 16150 Kubang Kerian, Kelantan, Malaysia; Tel: +6-0169303787;

Fax:+6-09-7647884; E-mail: mibrahim12@yahoo.com
There is much information regarding the health benefits of honey but a little information is available about the antioxidant properties of honey and its role against different types of diseases. The objective of this review is to summarize antioxidant properties of honey and its role against health disorder like cancer, cardiovascular diseases, inflammatory disorders, neurological degeneration, infectious diseases and aging.

\section{COMPOSITIONAL PROPERTIES OF HONEY}

Phytochemicals are one wide class of nutraceuticals found in plants which are extensively researched by scientists for their health-promoting potential. Honey has a wide range of phytochemicals including polyphenols which act as antioxidants. Polyphenols and phenolic acids found in the honey vary according to the geographical and climatic conditions. Some of them were reported as a specific marker for the botanical origin of the honey. Considerable differences in both composition and content of phenolic compounds have been found in different unifloral honeys [12]. Terpenes, benzyl alcohol, 3, 5-dimethoxy-4-hydroxybenzoic acid (syringic acid), methyl 3, 5-dimethoxy-4-hydroxybenzoate (methyl syringate), 3, 4, 5-trimethoxybenzoic acid, 2hydroxy-3- phenylpropionic acid, 2-hydroxybenzoic acid and 1,4-dihydroxybenzene are some of the phytochemicals ascribed for the antimicrobial activity of honey [13].

The color of the honey collected by the bees varies according to the floral source and its mineral content, which usually ranges from water white to dark amber. Flavor of the honey depends upon the color, generally the darker the honey the stronger the flavor and quality. It has been reported more than 300 unique varieties of honey depending upon the floral sources from United States alone. Honey mainly composed of sugars and water which accounts roughly $79.6 \%$ and $17.2 \%$, respectively. Major sugars of 
honey are levulose and dextrose which constitutes $38.19 \%$ and $31.28 \%$ correspondingly, remaining is the sucrose $1.3 \%$ and maltose $7.3 \%$. Honey minor constituents include acids $(0.57 \%)$, protein $(0.266 \%)$, nitrogen $(0.043 \%)$, amino acids $(0.1 \%)$, a little amount of minerals $(0.17 \%)$, and a number of other minute quantities of components like pigments, flavor and aroma substances, phenolics compounds, colloids, sugar alcohols and vitamins which all together accounts for the $2.1 \%$ of whole honey composition [14].

\section{FREE RADICAL AND ANTIOXIDANTS}

Although oxygen is vital to life, scientists are also finding that this essential element may contribute to human aging and illness. When oxygen is metabolized, cells form byproducts called "free radicals." Free radicals travel through the cell, disrupting the structure of other molecules and resulting in cellular damage. Such damage is believed to contribute to aging and various health problems. Antioxidants protect key cell components from damage by neutralizing the free radicals. Antioxidants that occur naturally in the body or are consumed through the diet may block damage to cells. However, over time, damaged cells can accumulate and lead to age-related diseases, researchers say [15].

In an effort to combat free-radical activity, scientists are studying the effects of increasing individuals' antioxidant levels through the diet and dietary supplements. Honey appears to act as an antioxidant in more ways than one. In the body, honey can take up free radicals and contribute to better health. When used in foods, the compounds produced when honey is heated can prevent rancidity in some products, particularly meats [16].

\section{ANTIOXIDANT PROPERTIES OF HONEY}

Although free radicals of oxygen are a natural byproduct of metabolism within the organism, they cause cellular damage and breakdown the structure of DNA. Exactly these processes cause premature aging. Antioxidants bind these dangerous molecules, preventing their harmful effects [17]. Unlike synthetic compounds, honey represents a natural product that does not carry side effects which can be harmful to health. Among the compounds found in honey; vitamin C, phenol compounds, catalase, peroxides, glucose oxydase enzymes have antioxidant properties. Honey also contains flavonoids and carotinoids. High levels of these indicators ensure a high level of antioxidants in honey. Antioxidant properties of honey act as an antidepressant during high emotional, physical and intellectual stress [17].

Various polyphenols are reported in honey. Some of the polyphenols of honey like Caffeic acid, Caffeic acid phenyl ester, Chrysin, Galangin, Quercetin, Acacetin, Kaempferol, Pinocembrin, Pinobanksin and Apigenin have evolved as promising pharmacological agents in treatment of cancer [17].

"Gram for gram, antioxidants in buckwheat honey equal those of fruits and vegetables," said Dr. May Berenbaum, head of the University of Illinois' entomology department. "It packs the antioxidant power of Vitamin C in a tomato." Researchers at the University of Illinois-Champaign/Urbana have identified the antioxidant values of 14 unifloral honeys. The antioxidative components of honey were compared to an ascorbic acid standard. The water-soluble antioxidant content of the honey samples varied more than 20 -fold, from a high value of $4.32 \times 10^{-3}$ eq for Illinois buckwheat honey to a low value of $21.3 \times 10^{-5}$ eq for California button sage honey [15].

Research showed a correlation between color and antioxidant capacity, with the darker honeys providing the highest levels of antioxidants. With antioxidant levels reaching $4.32 \times 10^{-3}$ meq., honey rivals those levels found in tomatoes $\left(2.83 \times 10^{-3} \mathrm{meq}\right)$ and sweet corn $\left(1.36 \times 10^{-3} \mathrm{meq}\right)$. Although honey by itself may not serve as a major source of dietary antioxidants, it demonstrates the potential for honey to play a role in providing antioxidants in a highly palatable form. Due to honey's pleasing taste, it may be more readily consumed by individuals reluctant to ingest plant-derived antioxidants. Certainly, compared to sucrose, which has no antioxidant value, honey can be a flavorful, supplementary source of antioxidants [17].

\section{BOTANICAL ORIGIN AND ANTIOXIDANT ACTIVI- TIES OF HONEY}

Honey has been found to contain significant antioxidant compounds including glucose oxidase, catalase, ascorbic acid, flavonoids, phenolic acids, carotenoid derivatives, organic acids, Maillard reaction products, amino acids and proteins [18-26]. The antioxidative activity of honey polyphenols can be measured in vitro by comparing the oxygen radical absorbance capacity (ORAC) with the total phenolics concentration (Table 1). There is a significant correlation between the antioxidant activity, the phenolic content of honey and the inhibition of the in vitro lipoprotein oxidation of human serum [27]. Furthermore, in a lipid peroxidation model system buckwheat honey showed a similar antioxidant activity as $1 \mathrm{mM} \alpha$-tocopherol [25]. The influence of honey ingestion on the antioxidative capacity of plasma was tested in two studies $[28,29]$. In the first one, the trial persons were given maize syrup or buckwheat honeys with a different antioxidant capacity in a dose of $1.5 \mathrm{~g} / \mathrm{kg}$ body weight. In comparison to the sugar control, honey caused an increase of both the antioxidant and the reducing serum capacity. In the second study humans received a diet supplemented with a daily honey serving of $1.2 \mathrm{~g} / \mathrm{kg}$ body weight. Honey increased the body antioxidant agents: blood vitamin C concentration by $47 \%, \beta$-carotene by $3 \%$, uric acid by $12 \%$, and glutathione reductase by $7 \%$ [29]. It should be borne in mind that the antioxidant activity depends on the botanical origin of honey and varies to a great extent in honeys from different botanical sources [30-32].

\section{ANTIOXIDANT IN HONEY ADDS HEALTH BENEFITS}

Departments of Nutrition and Internal Medicine at the University of California and National Honey Board, showed that free radicals and reactive oxygen species (ROS) have been implicated in contributing to the processes of aging and disease [34]. Humans protect themselves from these damaging compounds, in part, by absorbing antioxidants from high-antioxidant foods. This report describes the effects of consuming $1.5 \mathrm{~g} / \mathrm{kg}$ body weight of corn syrup or buckwheat honey on the antioxidant and reducing capacities of plasma in healthy human adults. The corn syrup treatment contained 
Table 1. Antioxidative Activity (ORAC) and Total Phenol Content of Different Unifloral Honeys [33]

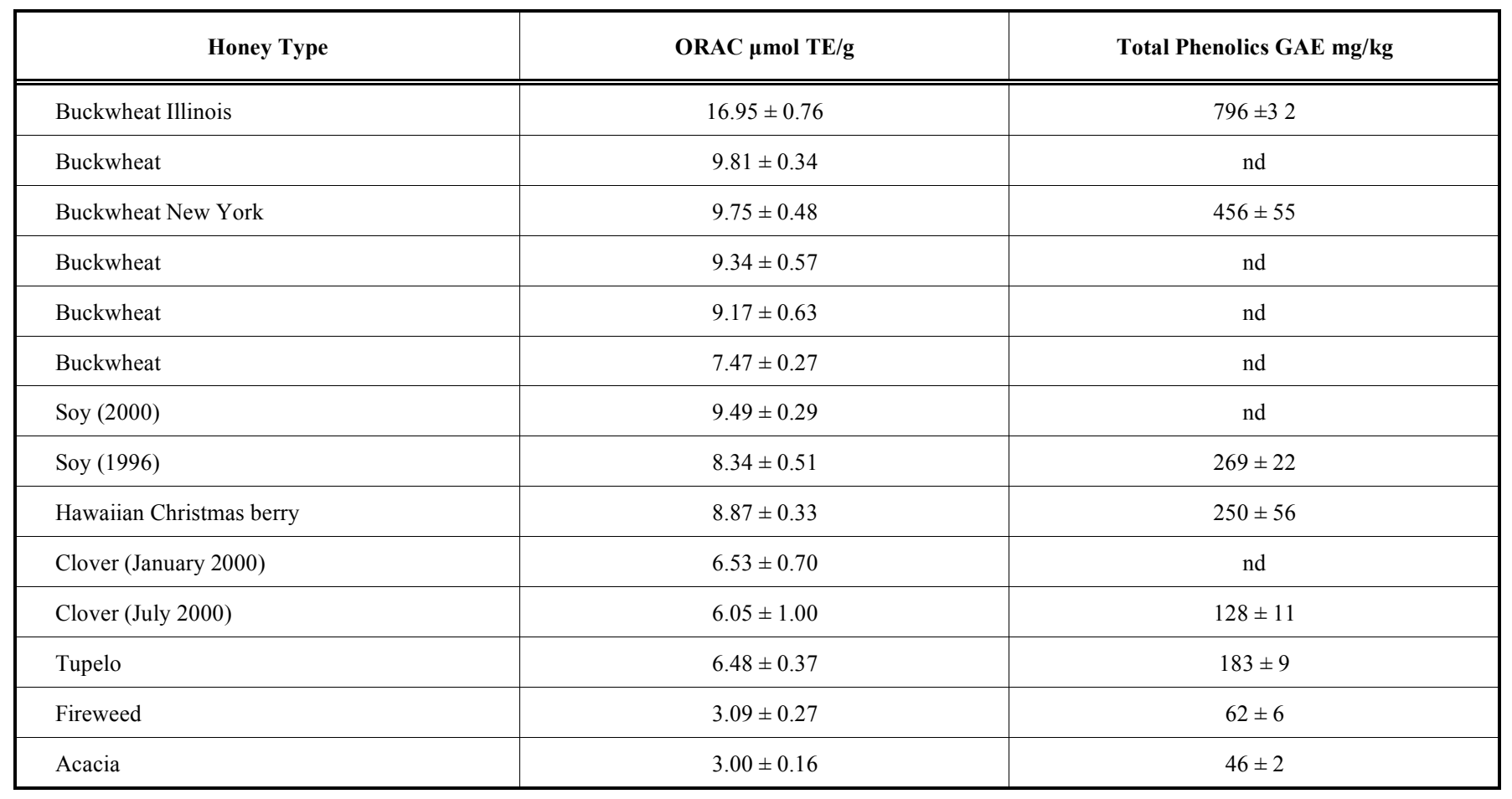

ORAC $=$ Oxygen radical absorbance capacity,

$\mathrm{TE}=$ Trolox equivalent, $\mathrm{GAE}=$ gallic acid equivalent, $\mathrm{nd}=$ not determined.

0.21 ( $0.06 \mathrm{mg}$ of phenolic antioxidants per gram, and the two buckwheat honey treatments contained $0.79(0.02$ and 1.71 $(0.21 \mathrm{mg}$ of phenolic antioxidants per gram. Following consumption of the two honey treatments, plasma total-phenolic content increased $(\mathrm{P}<0.05)$ as did plasma antioxidant and reducing capacities $(\mathrm{P}<0.05)$. These data support the concept that phenolic antioxidants from processed honey are bioavailable, and that they increase antioxidant activity of plasma. It can be speculated that these compounds may augment defenses against oxidative stress and that they might be able to protect humans from oxidative stress. Given that the average sweetener intake by humans is estimated to be in excess of $70 \mathrm{~kg}$ per year, the substitution of honey in some foods for traditional sweeteners could result in an enhanced antioxidant defense system in healthy adults [34].

Beretta et al. demonstrated the protective activity of a honey of multifloral origin, standardized for total antioxidant power and analytically profiled (HPLC-MS) in antioxidants, in a cultured endothelial cell line (EA.hy926) subjected to oxidative stress. Cumene hydroperoxide $(\mathrm{CuOOH})$ was used as free radical promoter. Native honey $(1 \% \mathrm{w} / \mathrm{v} \mathrm{pH} 7.4$, $10(6)$ cells) showed strong quenching activity against lipophilic cumoxyl and cumoperoxyl radicals, with significant suppression/prevention of cell damage, complete inhibition of cell membrane oxidation, of intracellular ROS production and recovery of intracellular GSH. Experiments with endothelial cells fortified with the isolated fraction from native honey enriched in antioxidants, exposed to peroxyl radicals from 1,1-diphenyl-2-picrylhydrazyl (AAPH, $10 \mathrm{mM}$ ) and to hydrogen peroxide $\left(\mathrm{H}_{2} \mathrm{O}_{2}, 50-100\right.$ microM), indicated that phenolic acids and flavonoids were the main causes of the protective effect. They suggested that, through the synergistic action of its antioxidants, honey by reducing and removing ROS, may lower the risks and effects of acute and chronic free radical induced pathologies in vivo [35].

\section{ROLE OF ANTIOXIDANT IN PREVENTING CANCER}

Chemoprevention utilizes appropriate pharmacological agents [3, 4] or of dietary agents, consumed in diverse forms like macronutrients, micronutrients, or nonnutritive phytochemicals. Various polyphenols, which have antioxidant properties, are reported in honey. Some of the polyphenols of honey like Caffeic acid, Caffeic acid phenyl ester, Chrysin, Galangin, Quercetin, Acacetin, Kaempferol, Pinocembrin, Pinobanksin and Apigenin have evolved as promising pharmacological agents in treatment of cancer [15].

Mok-Ryeon Ahn et al. showed that acacetin, apigenin, artepillin C, caffeic acid phenethyl ester, chrysin, $p$-coumaric acid, galangin, kaempferol, pinocembrin, and quercetin have antioxidant activity. These components were evaluated for their antioxidant activities by 1,1-diphenyl-2-picrylhydrazyl (DPPH) free radical-scavenging and ferric reducing/ antioxidant power (FRAP) assays. Furthermore, the effects of these components were tested on in vitro models of angiogenesis, tube formation and growth of human umbilical vein endothelial cells (HUVECs). Caffeic acid phenethyl ester, and quercetin, possessed strong inhibitory effects on tube formation and on endothelial cell proliferation and, coincidentally, showed strong antioxidant activity. Artepillin $\mathrm{C}$, galangin, and kaempferol also possessed strong antiangiogenic and antioxidant activities to a slightly less degree. In 
contrast, acacetin, apigenin, and pinocembrin possessed a considerable degree of antiangiogenic activities, although they showed very low antioxidant activities. These results demonstrated that artepillin $\mathrm{C}$, caffeic acid phenethyl ester, galangin, kaempferol, and quercetin might represent a new class of dietary-derived antioxidative compounds with antiangiogenic activities. These components may have the potential to be developed into pharmaceutical drugs for the treatment of angiogenesis-dependent human diseases such as tumors [36].

Caffeic acid has been reported as a carcinogen in initial studies, but the same caffeic acid along with combination of other antioxidant has been shown to suppress colon tumors in rats. Natarajan et al. demonstrated that Caffeic acid phenyl ester (CAPE) is known to have antimitogenic, anticarcinogenic, antiinflammatory and immunomodulatory properties [37]. CAPE's antiinflammatory and anticancer property has also been shown to protect skin cells when exposed to ultra-violet radiation and UVB radiation [38].

Weng et al. showed that the growth inhibitory effect of chrysin in C6 glioma cells was either though activating p38MAPK which leads to the accumulation of $\mathrm{p} 21 \mathrm{Waf} 1 / \mathrm{Cip} 1$ protein or mediating the inhibition of proteasome activity [39]. In another study by Woo et al. it has been elucidated that chrysin induces apoptosis in association with the activation of caspase- 3 and Akt signal pathway, that plays a crucial role in chrysin-induced apoptosis in U937 cells [40]. Galangin expressed antiproliferative effect on HL-60 cells on dose dependent manner and also induced DNA fragmentation without loss of membrane integrity [41].

Quercetin also inhibited the HL-60 cell proliferation in association with the inhibition of cytosolic Protein Kinase C (PKC) and membrane Tyrosine Protein Kinase (TPK) in vitro [42]. It has been reported that quercetin in low concentration promoted cell proliferation of A-549 cells, whereas in higher concentration it inhibited cell proliferation and survival [43]. Further quercetin exerted antiproliferative effect against glioma and breast cancer cells [44-46].

Acacetin, another important flavanoid inhibited the proliferation of A549 cells, induced apoptosis and blocked the cell cycle progression at G1 phase. It also improved the expression of p53 and Fas ligands [47]. In another study, it has been shown to inhibit HepG2 cell proliferation and provoke apoptosis by enhancing the p53 and Fas ligands as in the case of A549 cells [48]. Kaempferol induced apoptosis in H460 cells which was accompanied by significant DNA condensation and increasing ATP levels. It also changed the expression of Caspase 3 and Apoptosis Inducing Factor (AIF) levels [49]. Bestwick et al. reported recently that kaempferol growth inhibitory effect on HL-60 leukemia cells is due to heterogeneous response mainly dominated by cell cycle alternation although some degree of cytotoxicity results from apoptotic as well as nonapoptotic process [50]. Pinocembrin induced loss of mitochondrial membrane potential (MMP) with subsequent release of cytochrome $\mathrm{c}$ and processing of caspase-9 and -3 in colon cancer cell line HCT 116 [51]. Apigenin exerted antiproliferative effect against colon, breast, cervical, neuroblastoma and liver cancer cell lines [52-56].

\section{ANTIOXIDANT AND CARDIOVASCULAR DIS- EASES}

Rakha et al. showed that induction of hyperadrenergic activity was experimentally achieved in urethaneanesthetized rats using epinephrine (adrenaline). Acute administration of epinephrine (100 microg/kg) for 2 hours induced several cardiac disorders and vasomotor dysfunction. Pretreatment with natural wild honey $(5 \mathrm{~g} / \mathrm{kg})$ for 1 hour prior to the injection with epinephrine (100 mug/kg) protected the anesthetized normal rats from the incidence of epinephrine-induced cardiac disorders and vasomotor dysfunction. Moreover, posttreatment with natural wild honey $(5 \mathrm{~g} / \mathrm{kg})$ following the injection with epinephrine (100 mi$\mathrm{crog} / \mathrm{kg}$ ) for 1 hour showed several ameliorative outcomes to the electrocardiographic parameters and vasomotor dysfunction of anesthetized stressed rats. Furthermore, natural wild honey preserved the positive inotropic effect of epinephrine in both cases. Also, the total antioxidant capacity (AOC) of natural wild honey was found to be very pronounced. Levels of both reduced glutathione and ascorbic acid (vitamin C) were considered relatively high in natural wild honey. Activity of superoxide dismutase (SOD) was also high, whereas catalase activity was relatively low, especially when compared to the value of SOD activity. It would appear from the results of the present study that natural wild honey may exert its cardioprotective and therapeutic effects against epinephrine-induced cardiac disorders and vasomotor dysfunction directly, via its very pronounced total AOC and its great wealth of both enzymatic and nonenzymatic antioxidants involved in cardiovascular defense mechanisms, besides its substantial quantities of mineral elements such as magnesium, sodium, and chlorine, and/or indirectly, via the enhancement of the endothelium-derived relaxing factor nitric oxide release through the influence of ascorbic acid (vitamin C) [57].

Nagyova et al. also showed that antioxidants and antioxidant enzymes protect living organisms against the attack of reactive oxygen species. An adequate daily intake of the individual antioxidants is therefore important to prevent the cells against oxidative damage. They investigated the effect of a modest dietary supplementation with a mixture of antioxidant nutrients (100 mg vitamin E, $100 \mathrm{mg}$ vitamin C,6 mg beta-carotene and 50 microg of selenium per day) for 3 months on the plasma antioxidant capacity and indices of oxidative stress. Two groups of middle-age men were selected: group 1 with survivors of myocardial infarction (MI), and group 2 with clinically normal controls. The values of total antioxidant capacity of plasma (FRAP) significantly increased after supplementation with antioxidants in the both groups. Markers of in vivo lipid peroxidation, plasma malondialdehyde (MDA) and conjugated diene (CD) levels significantly decreased in the both supplemented groups. MDA and CD values were significantly higher at baseline in the group of survivors of myocardial infarction when compared with the group of healthy men. The results demonstrate that short-term and modest supplementation with a mixture of antioxidant nutrients improves antioxidative capacity and reduces products of lipid peroxidation in plasma. Since a more pronounced effect was observed within the group of survivors of myocardial infarction, a recommenda- 
tion of antioxidant supplements seems appropriate for patients with a history of cardiovascular disease [58].

Yochum et al. demonstrated that Flavonoids, a group of phenolic compounds found in fruits and vegetables, are known to have antioxidant properties. They prevent low density lipoprotein oxidation in vitro and thus may play a role in the prevention of coronary heart disease (CHD). In 1986, in a prospective study of 34,492 postmenopausal women in Iowa, the authors examined the association of flavonoid intake with CHD and stroke mortality. Over 10 years of follow-up, 438 deaths from CHD and 131 deaths from stroke were documented. Total flavonoid intake was associated with a decreased risk of CHD death after adjusting for age and energy intake ( $p$ for trend $=0.04$ ). This association was attenuated after multivariate adjustment. However, decreased risk was seen in each category of intake compared with the lowest. Relative risks and 95\% confidence intervals of CHD death from lowest to highest intake category were 1.0, 0.67 (95\% confidence interval $(\mathrm{Cl}) \quad 0.49-0.92), 0.56(95 \% \mathrm{Cl}$ $0.39-0.79), 0.86$ (95\% Cl 0.63-1.18), and 0.62 (95\% $\mathrm{Cl} 0.44-$ $0.87)$. There was no association between total flavonoid intake and stroke mortality $(\mathrm{p}$ for trend $=0.83$ ). Of the foods that contributed the most to flavonoid intake in this cohort, only broccoli was strongly associated with reduced risk of CHD death. The data of this study suggest that flavonoid intake may reduce risk of death from CHD in postmenopausal women [59].

Xia et al. showed that the endothelium-derived vasoconstrictor endothelin-1 is increased after cardiopulmonary bypass in children with congenital heart defects. This study determines whether antioxidant therapy with Salvia miltiorrhiza injection, an herb extract containing phenolic compounds, prevents the postoperative increase of endothelin-1. The relationship between endothelin-1 and the endotheliumderived prostacyclin (prostaglandin I2) and thromboxane A2 postoperatively is also investigated. Twenty children with congenital heart defects and pulmonary hypertension were randomly assigned to group A (placebo control, $n=10$ ) or $B$ $(200 \mathrm{mg} / \mathrm{kg}$ Salvia miltiorrhiza intravenously after anesthesia induction and at the time of rewarming, respectively; $n=10$ ) before cardiac surgery. Central venous blood samples were taken before operation $\left(\mathrm{T}_{0}\right), 10\left(\mathrm{~T}_{1}\right)$ and 30 minutes $\left(\mathrm{T}_{2}\right)$ after starting cardiopulmonary bypass, $10\left(\mathrm{~T}_{3}\right)$ and 30 minutes $\left(\mathrm{T}_{4}\right)$ after aortic declamping, and 30 minutes $\left(\mathrm{T}_{5}\right)$ and 24 hours $\left(\mathrm{T}_{6}\right)$ after termination of cardiopulmonary bypass. Plasma lipid peroxidation product malondialdehyde, myocardial specific creatine kinase-MB activity, thromboxane B2, and 6-keto-prostaglandin $F_{1 \alpha}$ (stable metabolites of thromboxane A2 and prostaglandin I2) were measured. Malondialdehyde increased significantly at $T_{1}$ in group $A$ and remained significantly higher than in group $\mathrm{B}$ thereafter $(P<.05)$. Malondialdehyde in group B did not significantly increase over time. At $\mathrm{T}_{5}$, plasma creatine kinase-MB, thromboxane B2, and endothelin-1 in group B were lower than in group A $(\mathrm{P}<.05)$; malondialdehyde correlated significantly with creatine kinase-MB $(r=0.71, \mathrm{P}=.0005)$. At $\mathrm{T}_{6}$, endothelin-1 negatively correlated with the 6-ketoprostaglandin $\mathrm{F}_{1 \alpha} /$ thromboxane $\mathrm{B} 2$ ratio $(r=0.64, \mathrm{P}=.0025)$. This study concluded that antioxidant therapy reduces myocardial damage and attenuates postoperative vasoactive mediator imbalance [60].
Some of the polyphenols of honey like Quercetin, Caffeic acid phenyl ester (CAPE), Galangin, Acacetin, Kaempferol and Pinocembrin have been reported as promising pharmaceutical drugs in the treatment of cardiovascular diseases.

\section{QUERCETIN}

Angiotensin II (Ang II) induces vascular smooth muscle cell (VSMC) hypertrophy, which results in various cardiovascular diseases. Ang II-induced cellular events have been implicated, in part, in the activation of mitogen-activated protein (MAP) kinases. Although it has been proposed that daily intake of bioflavonoids belonging to polyphenols reduces the incidence of ischemic heart diseases (known as "French paradox"), the precise mechanisms of efficacy have not been elucidated. Thus, they hypothesized that bioflavonoids may affect Ang II-induced MAP kinase activation in cultured rat aortic smooth muscle cells (RASMC). Our findings showed that Ang II stimulated rapid and significant activation of extracellular signal-regulated kinase (ERK) $1 / 2$, c-Jun N-terminal kinase (JNK), and p38 in RASMC. Ang IIinduced JNK activation was inhibited by 3,3',4',5,7pentahydroxyflavone (quercetin), a major bioflavonoid in foods of plant origin, whereas ERK $1 / 2$ and p38 activation by Ang II were not affected by quercetin. Ang II caused a rapid tyrosine phosphorylation of Src homology and collagen (Shc), which was inhibited by quercetin. Quercetin also inhibited Ang II-induced Shc.p85 association and subsequent activation of phosphatidylinositol 3-kinase (PI3-K)/Akt pathway in RASMC. Furthermore, LY294002, a PI3-K inhibitor and a quercetin derivative, inhibited Ang II-induced JNK activation as well as Akt phosphorylation. Finally, Ang II-induced [(3)H] leucine incorporation was abolished by both quercetin and LY294002. These findings suggest that the preventing effect of quercetin on Ang II-induced VSMC hypertrophy are attributable, in part, to its inhibitory effect on Shc- and PI3-K-dependent JNK activation in VSMC. Thus, inhibition of JNK by quercetin may imply its usefulness for the treatment of cardiovascular diseases relevant to VSMC growth [61].

Several studies have found that chronic treatment with the dietary flavonoid quercetin lowers blood pressure and restores endothelial dysfunction in hypertensive animal models. Male SHR and Wistar-Kyoto (WKY) rats (5 weeks old) were treated with quercetin $(10 \mathrm{mg} / \mathrm{kg})$ or vehicle for 13 weeks. Changes in vascular expression of eNOS, caveolin-1 and p47 were analysed by Western blot, eNOS activity by conversion of $[\mathrm{H}]$ arginine to $\mathrm{L}-[\mathrm{H}]$ citrulline, and NADPH oxidase activity by NADPH-enhanced chemoluminescence of lucigenin. In SHR, quercetin reduced the increase in blood pressure and heart rate and enhanced the endotheliumdependent aortic vasodilation induced by acetylcholine, but had no effect on the endothelium-independent response induced by nitroprusside. However, quercetin had no effect on endothelium-dependent vasoconstriction and aortic thromboxane B2 production. Compared to WKY, SHR showed upregulated eNOS and p47 protein expression, downregulated caveolin-1 expression, increased NADPH-induced superoxide production but, paradoxically, eNOS activity was reduced. Chronic quercetin treatment prevented all these changes in SHR. In WKY, quercetin had no effect on blood 
pressure, endothelial function or the expression or activity of the proteins analysed. Enhanced eNOS activity and decreased NADPH oxidase-mediated superoxide anion $\left(\mathrm{O}_{2}\right)$ generation associated with reduced p47 expression appear to be essential mechanisms for the improvement of endothelial function and the antihypertensive effects of chronic quercetin [62].

Carlstrom et al. showed that diets high in quercetin may decrease the risk of developing cardiovascular disease. They tested whether quercetin delays or reduces the severity of hypertension, vascular dysfunction, or cardiac hypertrophy in the spontaneously hypertensive rat (SHR). Normotensive, 5-wk-old SHR consumed standard (n 1/4 18) or quercetinsupplemented diet (1.5 g quercetin/kg diet, $\mathrm{n} 1 / 4$ 22, SHR-Q) for 5 or 11 wk. Wistar Kyoto rats (WKY, n 1/4 19), fed a standard diet, served as controls. At $16 \mathrm{wk}$, plasma quercetin, measured by HPLC, was $2.0960 .33 \mathrm{mmol} / \mathrm{L}$ in SHR$\mathrm{Q}$ and below assay detection limits in SHR and WKY rats. At 10 and 16 wk of age, arterial blood pressure and heart weight:body weight were not different between SHR and SHR-Q. At $16 \mathrm{wk}$, cardiac function (echocardiography), vascular morphology (hematoxylin and eosin staining of aortae), and resistance and conductance vessel reactivity (wire myography) was unchanged in SHR vs. SHR-Q. Thus, a quercetin supplemented diet does not delay the onset or lessen the severity of cardiovascular complications that develop in SHR. These findings contrast with previous reports of cardiovascular protection when quercetin was delivered via oral gavage. To determine whether the efficacy of quercetin depends on its method of delivery, 15-wk-old SHR were given quercetin (10 $\mathrm{mg} / \mathrm{kg}$ ) once daily via oral gavage for 4 consecutive days. Arterial blood pressure $(\mathrm{mm} \mathrm{Hg})$ was lower in gavaged SHR (148 65$)$ than in SHR-Q (162 62 , P , 0.02) and SHR (168 63 , P , 0.001). These data suggest that mode of delivery is a critical determinant in whether quercetin provides cardiovascular benefits [63].

In another study, the effects of an oral daily dose (10 mg $\mathrm{kg} 71$ ) of the avonoid quercetin for 5 weeks in spontaneously hypertensive (SHR) and normotensive Wistar Kyoto rats (WKY) were analysed. Quercetin induced a significant reduction in systolic (718\%), diastolic (723\%) and mean (721\%) arterial blood pressure and heart rate $(712 \%)$ in SHR but not in WKY rats. The left ventricular weight index and the kidney weight index in vehicle-treated SHR were significantly greater than in control WKY and these parameters were significantly reduced in quercetin-treated SHR in parallel with the reduction in systolic blood pressure. Quercetin had no effect on the vasodilator responses to sodium nitroprusside or to the vasoconstrictor responses to noradrenaline or $\mathrm{KCl}$ but enhanced the endothelium-dependent relaxation to acetylcholine $(\mathrm{Emax}=58+5 \%$ vs $78+5 \%$, P50.01) in isolated aortae. The $24 \mathrm{~h}$ urinary isoprostane F2a excretion and the plasma malonyldialdehyde (MDA) levels in SHR rats were increased as compared to WKY rats. However, in quercetin-treated SHR rats both parameters were similar to those of vehicle-treated WKY. These data demonstrate that quercetin reduces the elevated blood pressure, the cardiac and renal hypertrophy and the functional vascular changes in SHR rats without effect on WKY. These effects were associ- ated with a reduced oxidant status due to the antioxidant properties of the drug [64].

Epidemiological studies reported that quercetin, an antioxidant flavonol is associated with reduced risk of coronary heart disease and stroke. Quercetin supplementation also reduces blood pressure in hypertensive rodents. The efficacy of quercetin supplementation to lower blood pressure in hypertensive humans has never been evaluated. They tested the hypothesis that quercetin supplementation reduces blood pressure in hypertensive patients and then determined whether the antihypertensive effect of quercetin is associated with reductions in systemic oxidant stress. Men and women with prehypertension (n 1/4 19) and stage 1 hypertension (n $1 / 4$ 22) were enrolled in a randomized, double-blind, placebocontrolled, crossover study to test the efficacy of $730 \mathrm{mg}$ quercetin/d for $28 \mathrm{~d}$ vs. placebo. Blood pressure $(\mathrm{mm} \mathrm{Hg}$, systolic/diastolic) at enrollment was $13762 / 8661$ in prehypertensives and $14862 / 9661$ in stage 1 hypertensive subjects. Blood pressure was not altered in prehypertensive patients after quercetin supplementation. In contrast, reductions in $(\mathrm{P}, 0.01)$ systolic $(2762 \mathrm{mmHg})$, diastolic $(256$ $2 \mathrm{mmHg})$, andmean arterial pressures $(2562 \mathrm{~mm} \mathrm{Hg})$ were observed in stage 1 hypertensive patients after quercetin treatment. However, indices of oxidant stress measured in the plasma and urinewere not affected by quercetin. These data showed that quercetin supplementation reduces blood pressure in hypertensive subjects. Contrary to animal-based studies, there was no quercetin-evoked reduction in systemic markers of oxidative stress [65].

\section{ACACETIN}

Gui-Rong et al. investigated whether the natural flavone acacetin would be an atrium-selective anti-atrial fibrillation agent. The effects of acacetin on human atrial ultrarapid delayed rectifier $\mathrm{K}^{+}$current $\left(I_{\mathrm{Kur}}\right)$ and other cardiac ionic currents were studied with a whole-cell patch technique. Acacetin suppressed $I_{\mathrm{Kur}}$ and the transient outward $\mathrm{K}^{+}$current $\left(\mathrm{IC}_{50} 3.2\right.$ and $9.2 \mu \mathrm{mol} / \mathrm{L}$, respectively) and prolonged action potential duration in human atrial myocytes. The compound blocked the acetylcholine-activated $\mathrm{K}^{+}$current; however, it had no effect on the $\mathrm{Na}^{+}$current, L-type $\mathrm{Ca}^{2+}$ current, or inward-rectifier $\mathrm{K}^{+}$current in guinea pig cardiac myocytes. Although acacetin caused a weak reduction in the hERG and hKCNQ1/hKCNE1 channels stably expressed in HEK 293 cells, it did not prolong the corrected QT interval in rabbit hearts. In anesthetized dogs, acacetin $(5 \mathrm{mg} / \mathrm{kg})$ prolonged the atrial effective refractory period in both the right and left atria 1 to 4 hours after intraduodenal administration without prolongation of the corrected QT interval, whereas sotalol at $5 \mathrm{mg} / \mathrm{kg}$ prolonged both the atrial effective refractory period and the corrected QT interval. Acacetin prevented atrial fibrillation (AF) induction at doses of $2.5 \mathrm{mg} / \mathrm{kg}(50 \%), 5 \mathrm{mg} / \mathrm{kg}(85.7 \%)$, and $10 \mathrm{mg} / \mathrm{kg}$ $(85.7 \%)$. Sotalol $5 \mathrm{mg} / \mathrm{kg}$ also prevented AF induction $(60 \%)$. The study demonstrated that the natural compound acacetin is an atrium-selective agent that prolongs the atrial effective refractory period without prolonging the corrected QT interval and effectively prevents AF in anesthetized dogs after intraduodenal administration. These results indicated that oral acacetin is a promising atrium-selective agent for the treatment of AF [66]. 


\section{CAFFEIC ACID}

Caffeic acid phenethyl ester (CAPE) is a phenolic active component of propolis of honeybee hives and reduces heart rate and blood pressure in rats. Iraz et al. investigated the role of vagal activity and atropine blockage on the bradycardic and hypotensive effects of CAPE in rats. The rats were divided into five groups $(\mathrm{n}=8)$. Saline and vehicle $(10 \%$ ethanol) of CAPE were given to the first and second groups, respectively. Group 3 was treated with $5 \mathrm{mg} / \mathrm{kg}$ CAPE. Group 4 bivagotomized and treated with $5 \mathrm{mg} / \mathrm{kg}$ CAPE. Group 5 treated with atropine $(5 \mathrm{microg} / \mathrm{microL} / \mathrm{min})$ continuously and treated with CAPE. The electrophysiological monitoring was done for each experiment under urethane anesthetize. As a result, CAPE caused intense and transient bradycardia and hypotension. Vagotomy completely abolished bradycardia occurred via CAPE injection; however atropine attenuated bradycardic effects of CAPE. On the other hand, hypotensive effect of CAPE was affected from neither bilateral vagotomy nor atropine treatment. It was thought that CAPE may exert its effects on heart rate via a central parasympathetic control mechanism, but not on central parasympathetic blood pressure control system [67].

\section{KAEMPFEROL}

The vascular effects of kaempferol were investigated in isolated porcine coronary artery rings. U46619 (9,11dideoxy- $9 \alpha, 11 \alpha$-methanoepoxy prostaglandin $\mathrm{F} 2 \alpha, 30 \mathrm{nM}$ ) was used to contract porcine coronary arterial rings. Concentration relaxation curve of kaempferol $(1 \mathrm{nM}-100 \mu \mathrm{M})$ was constructed and kaempferol demonstrated significant relaxation at high concentrations. At low concentration with no significant effect on relaxation, kaempferol $(10 \mu \mathrm{M})$ enhanced relaxation produced by bradykinin, the calcium ionophore A23187, isoproterenol and sodium nitroprusside in endothelium-intact porcine coronary arteries. In endothelium-disrupt rings, kaempferol $(10 \mu \mathrm{M})$ also enhanced the relaxation caused by isoproterenol, sodium nitroprusside, levcromakalim and nifedipine. On the other hand, antioxidant agents did not affect bradykinin-induced relaxation or the enhancement effect of kaempferol. It was concluded that a low concentration of kaempferol $(10 \mu \mathrm{M})$, devoid of significant vascular effect, has the ability to enhance endothelium-dependent and endothelium-independent relaxations. This action of kaempferol is unrelated to its antioxidant property [68].

In a study examined whether or not the ER stress and Bcl-2 proteins are linked to the protective effect of kaempferol, a phytoestrogen, on ischemia-reperfusion (I/R)induced cardiac damage. In order to determine if kaempferol modifies the I/R-induced response in $\mathrm{H} 9 \mathrm{c} 2$ cardiac muscle cells, the cells were exposed to kaempferol followed by ischemia $12 \mathrm{~h} /$ reperfusion $4 \mathrm{~h}$. kaempferol had a protective effect on the apoptosis induced by $I / R$ in the cardiac muscle cells. The kaempferol treatment significantly increased the expression level of the anti-apoptotic protein, Bcl-2, but decreased the level of the pro-apoptotic protein, bax. Kaempferol down-regulated the expressions of the endoplasmic reticulum (ER) stress proteins, GRP78, ATF-6alpha, XBP-2, IRE1-alpha, phosphor-eIF-2alpha and CHOP. In $e x$ vivo-Langendorff experiment, the kaempferol treatment regulated the expression of ER stress proteins-CHOP and
GRP78. The kaempferol also improved the post-ischemic LVEDP and LVDP significantly after 20, 30, 40 and $50 \mathrm{~min}$ of reperfusion compared with the untreated control hearts, which showed that kaempferol offers protection against I/Rassociated cardiac dysfunction [69].

\section{GALANGIN}

Effect on prevention of heart disease and lipid peroxidation can prevent heart disease and has anti-oxidative effect on endothelial tissues. Therefore, it helps preserve other protective antioxidants such as vitamin E, vitamin $\mathrm{C}$, and other flavonoids, and also can prevent lipid peroxidation [70].

\section{ANTIOXIDANT AND NEURAL DISEASES}

Flavonoids have been intensively studied on their pharmacological activities such as anti-cancer, anti-oxidant and anti-inflammation. However, little is known about their neuroprotective effects. Recent studies suggest that inflammation mediated by microglia may play a role in neurodegenerative diseases. In a study, they evaluated the antiinflammatory effect of various flavonoid compounds by using BV-2, a murine microglia cell line. Of the compounds that were evaluated, apigenin inhibited the production of nitric oxide and prostaglandin E2 by suppressing the expression of inducible nitric oxide synthase and cyclooxygenase-2 protein, respectively. Moreover, apigenin suppressed p38 mitogen-activated protein kinase (MAPK), c-Jun N-terminal kinase (JNK) phosphorylation without affecting the activity of extracellular signal-regulated kinase (ERK). Apigenin was also found to protect neuronal cells from injury in middle cerebral artery occlusion [71].

Using the patch-clamp technique, Losi et al. have studied the modulation of ionotropic $\gamma$-aminobutyric acid (GABA) and glutamate neurotransmission by apigenin, a flavonoid with sedative and antidepressant activity. Apigenin reversibly reduced GABA-evoked currents mediated by a1h2g2 receptors expressed in HEK293 cells. Amplitude and frequency of spontaneous postsynaptic inhibitory currents (sIPSCs) mediated by GABAA receptors were also decreased by apigenin in cultured cortical neurons. The flavonoid was almost inactive on a-amino-3-hydroxy-5methylisoxazolepropionic acid (AMPA) mediated currents while it reduced N-methyl-d-aspartate (NMDA) receptor mediated responses with a half maximal inhibiting concentration (IC50) of 10 AM. The flavonoid inhibited also peak amplitude and frequency of spontaneous postsynaptic excitatory currents (sEPSCs). Finally, apigenin is neuroprotective against glutamate-induced neurotoxicity in cerebellar and cortical neurons in culture. This data reveal the antagonistic effect of apigenin on GABA and NMDA channels. While the inhibition on GABA receptor cannot explain the effects of the drug in vivo this data on NMDA channels reveal a new target of apigenin. A reduction of the network excitability could thus account for the sedative effects. Furthermore, this data suggest a potential neuroprotective activity of apigenin [72].

\section{PINOCEMBRIN}

Pinocembrin is one of the flavonoids at the highest concentration in propolis. In a study has investigated the neuro- 
protective effect of pinocembrin on ischemia/reperfusion and ischemia/reperfusion-like insults. Protection by pinocembrin was studied at the in vivo level using a model of middle cerebral artery occlusion and reperfusion in rats. Pinocembrin was administrated at the start of reperfusion. Pinocembrin markedly increased rat viability, reduced infarct volumes and neurological deficit scores in all treatment groups. Primary cortical neuronal cultures were subjected to oxygenglucose deprivation/reoxygenation, a model of ischemia/ reperfusion-like injury, and treated with pinocembrin at the start of reoxygenation. Neuronal survival rates were increased, LDH release was decreased and both neurite length and apoptosis were alleviated when pinocembrin was present during reoxygenation, and this protection was associated with the reduction of reactive oxygen species, nitric oxide and neuronal nitric oxide synthase (nNOS) and inducible NOS (iNOS), and an increase of glutathione. Moreover, DNA laddering was decreased in treatment groups of pinocembrin. Caspase-3 protein was down-regulated and PARP degradation was alleviated after pinocembrin treatments. Our results suggest that pinocembrin may be a novel therapeutic strategy to reduce cerebral ischemia/reperfusion injury, and may act by the anti-oxidative and anti-apoptotic effects [73].

To study the effects of pinocembrin, a natural compound extracted from propolis, on cognitive ability impaired by chronic cerebral hypoperfusion in rats, and if it did so, to investigate its effects on brain mitochondria. Rat chronic cerebral hypoperfusion was achieved by permanent bilateral common carotid arteries ligation, with regional cerebral blood flow evaluated. Cognitive ability was tested by Morris water maze task. Production of reactive oxygen species and origin targets including mitochondria membrane potential, respiratory chain complex I, complex III activities and mitochondria swelling degree were evaluated. Cytochrome oxidase was determined on its expression level by western blotting. Pinocembrin alleviated cognitive impairments in Morris water maze and decreased mitochondria reactive oxygen species production, in accordance with its improvements on complex I activity, membrane potential level, mitochondria swelling degree and cytochrome oxidase deficits. Pinocembrin could improve rat cognitive impairments induced by chronic cerebral hypoperfusion, contributed to its protections on brain mitochondria structure and function [74].

\section{ANTIMICROBIAL ACTIVITIES}

Phenolic compounds of dark and clear honeys from Trásos-Montes of Portugal were extracted with Amberlite XAD2 and evaluated for their antioxidant and antimicrobial activities. The antioxidant effect was studied using the in vitro test capacity of scavenge the 2,2-diphenyl-1-picryhydrazyl (DPPH) free radical and of reducing power of iron (III)/ ferricyanide complex. The antimicrobial activity was screened using three Gram-positive bacteria (Bacillus subtilis, Staphylococcus aureus, Staphylococcus lentus) and three Gram-negative bacteria (Pseudomonas aeruginosa, Klebsiella pneumoniae and Escherichia coli). In the biological assays, results showed that $S$. aureus were the most sensitive microrganisms and B. subtilis, S. lentus, $K$. pneumoniae and $E$. coli were each moderately sensitive to the antimicrobial activity of honey extracts. Nevertheless, no antimicrobial activity was observed in the test with $\mathrm{P}$. aeruginosa [75].

Wahdan performed a study to clarify the possible causes of the antimicrobial activity of honey. A sugar solution resembling honey in its high sugar content was made. The antimicrobial activities of both honey and this solution towards 21 types of bacteria and two types of fungi were examined. The results achieved by both were compared. The difference between them indicated the presence of antimicrobial substance(s) in honey. The kinds of antimicrobial substances (inhibines) in honey are discussed. Hydrogen peroxide is not the only inhibine in honey. In fact, inhibines in honey include many other substances. Two important classes of these inhibines are the flavonoids and the phenolic acids. Flavonoids have often been extracted from honey previously. In this study two phenolic acids (caffeic acid and ferulic acid) were extracted from honey for the first time [76].

Antimicrobial activity of honey has been attributed to hydrogen peroxide, which is produced by naturally occurring glucose oxidase, and phenolic compounds. Taormina et al. investigated honey from six floral sources for their inhibitory activity against Escherichia coli O157:H7, Salmonella typhimurium, Shigella sonnei, Listeria monocytogenes, Staphylococcus aureus, and Bacillus cereus. A disc assay revealed that development of zones of inhibition of growth depends on the type and concentration of honey, as well as the test pathogen. Growth of $B$. cereus was least affected. The inhibition of growth of $S$. sonnei, L. monocytogenes, and $S$. aureus in $25 \%$ solutions of honeys was reduced by treating solutions with catalase, indicating that hydrogen peroxide contributes to antimicrobial activity. Darker colored honeys were generally more inhibitory than light colored honey. Darker honeys also contained higher antioxidant power. Since antimicrobial activity of the darker colored test honeys was not eliminated by catalase treatment, non-peroxide components such as antioxidants may contribute to controlling the growth of some foodborne pathogens [77].

\section{WOUND HEALING}

Henriques et al. investigated both free radical production and the antioxidant potential of some honeys, properties which may have a role to play in wound healing. Free radical production and quenching of three honey types (manuka, antibacterial but non-peroxide-producing; pasture, antibacterial peroxide-producing; commercial heat processed, nonantibacterial) was investigated by electron paramagnetic resonance (EPR) spectroscopy; quenching was also examined using a superoxide quenching assay. All honeys tested had antioxidant potential, with manuka able to completely quench added radicals within 5 min of spiking. Only the peroxide-producing honey (pasture PS9) was found to form radicals on dilution. The ability to modulate production and quenching of free radicals may contribute to the demonstrated ability of some honeys to help in resolving the state of inflammation typifying chronic wounds [78].

\section{FOOD PROCESSING}

Honeys from various floral sources were analyzed by Rasmussen et al. to select for utilization as a sweetener and 
potential source of antioxidants in the formulation of a salad dressing. On the basis of various indicators of potential antioxidant effectiveness, such as the ORAC (oxygen radical absorbance capacity) assay and identification of phenolic profile carried out by HPLC analysis, clover and blueberry honeys were selected. Dressings were stored under accelerated conditions (37 degrees C) for six weeks and at ambient (23 degrees $\mathrm{C}$ ) and refrigeration (4 degrees $\mathrm{C}$ ) temperatures for one year. Salad dressings incorporating honey provided protection against oxidation to a degree similar to that of EDTA, as determined by peroxide value and p-anisidine value. This demonstrated the potential for honey to be used as a substitute for EDTA and sweetener (such as HFCS) in commercial salad dressings [79].

In another study of Mckibben and Engeseth, the antioxidant content of different varieties of honey was investigated spectrophotometrically and honey's effectiveness in reducing oxidation of ground poultry determined by monitoring thiobarbituric acid reactive substances (TBARS). Buckwheat honey $(5 \%, \mathrm{w} / \mathrm{w})$ reduced TBARS $\sim 70 \%$, whereas acacia honey reduced TBARS $\sim 34 \%$ at 3 days of storage at $4{ }^{\circ} \mathrm{C}$. In comparison to butylated hydroxytoluene and tocopherol $(0.02 \%$ of total fat), honey (at $5 \%$ of the weight of the meat) was much more effective at preventing oxidation. Honey has great potential as an antioxidant source and may result in greater acceptability of meat products and prevent negative health implications of oxidized meats [80].

\section{CONCLUSION}

It is clear that the antioxidants properties of honey are due to the presence of some antioxidant compounds such as Vitamin C, monophenolics, flavonoids, and polyphenolics. Although there is a wide spectrum of antioxidant types, Caffeic acid, Caffeic acid phenyl ester, Chrysin, Galangin, Quercetin, Acacetin, Kaempferol, Pinocembrin, Pinobanksin and Apigenin predominate in many honeys. These antioxidant compounds have a promising pharmacological agent for preventing cancer, cardiovascular diseases, inflammatory disorders, neurological degeneration, wound healing, infectious diseases and aging as well as it can be used as food preservatives.

\section{REFERENCES}

[1] National Honey Board, Honey and Bees; April 2007. Available from: http://www.honey.com/consumers/kids/beefacts.asp. [cited 2009 Sep 15]

[2] White JW. Composition of honey. In: Crane E, Ed. Honey, a comprehensive survey. London: Bee research Association and Chalfont St Peter, 1975: 157-206.

[3] Ferreres F, Ortiz A, Silva C, Viguera CG, Barbera'n FAT, Lorente FT. Flavonoids of "La Alcarria" honey. Z Lebensm Unters Forsch 1992; 194: 139-43.

[4] Andrade P, Ferreres F, Amaral MT. Analysis of honey phenolic acids by HPLC, its application to honey botanical characterization. J Liq Chromatogr Relat Technol 1997; 20: 2281-8.

[5] Tan ST, Wilkins AL, Holland PT, McGhie TK. Extractives from New Zealand unifloral honeys. 2. Degraded carotenoids and other substances from heather honey. J Agric Food Chem 1989; 37: 1217-21.

[6] Cherchi A, Spanedda L, Tuberoso C, Cabras P. Solid-phase extraction of high-performance liquid chromatographic determination of organic acids in honey. J Chromatogr 1994; 669: 59-64.

[7] White JW, Rudyj ON. The protein content of honey. J Apic Res 1978; 17: 234-8.
[8] Hertog MG, Feskens EJ, Hollman PC, Katan MB, Kromhout D. Dietary antioxidant flavonoids and risk of coronary heart disease: the Zutphen Elderly Study. Lancet 1993, 342: 1007-111.

[9] Bravo L. Polyphenols: chemistry, dietary sources, metabolism, and nutritional significance. Nutr Rev 1998; 56: 317-33.

[10] Renaud S, De Lorgeril M. Wine, alcohol, platelets and the French paradox for coronary heart disease. Lancet 1992; 339: 1523-6.

[11] Serafini M, Ghiselli A, Ferro-Luzzi A. Red wine, tea and antioxidants. Lancet 1994; 344: 626.

[12] Amiot MJ, Aubert S, Gonnet M, Tacchini M. Les compos'es ph'enoliques des miels: 'etude pr'eliminaire sur l'identifi cation et la quantifi cation par familles. Apidologie 1989; 20: 115-25.

[13] World Wide Wounds, Honey as a topical antibacterial agent for treatment of infected wounds; February 2002. Available from: http://www.worldwidewounds.com/2001/november/Molan/honeyas-topical-agent.html. [cited 2009 Aug 15].

[14] Todd FE, Vansell GH. Pollen grains in nectar and honey. J Econ Entomol 1942; 35: 728-31.

[15] National Honey Board "Food Technology Program" Available from: http://www.aaccnet.org/funcfood/content/releases/Honeyantioxidant.htm. [cited 2009 Sep 5].

[16] Bashkir Bee Honey, Antioxidant properties of honey. Available from: http://info-bashkirbeehoney.com/eigenschaften/eigenschaften2. html. [cited 2009 Aug 15].

[17] Jaganathan SK, Mandal M. Antiproliferative Effects of Honey and of Its Polyphenols: A Review. J Biomed Biotechnol 2009; 2009: 830616.

[18] Beretta G, Granata P, Ferrero M, Orioli M, Facino RM. Standardization of antioxidant properties of honey by a combination of spectrophotometric/fluorimetric assays and chemometrics. Anal Chim Acta 2005; 533: 185-91.

[19] D'Arcy BR. Antioxidants in Australian floral honeys -Identification of health enhancing nutrient components. RIRDC Publication 2005; No 05/040, 1 .

[20] Frankel S, Robinson GE, Berenbaum MR. Antioxidant capacity and correlated characteristics of 14 unifloral honeys. J Apic Res 1998; 37: 27-31.

[21] Aljadi AM, Kamaruddin MY. Evaluation of the phenolic contents and antioxidant capacities of two Malaysian floral honeys. Food Chem 2004; 85: 513-8.

[22] Inoue K, Murayarna S, Seshimo F, Takeba K, Yoshimura Y, Nakazawa $\mathrm{H}$. Identification of phenolic compound in manuka honey as specific superoxide anion radical scavenger using electron spin resonance (ESR) and liquid chromatography with coulometric array detection. J Sci Food Agric 2005; 85: 872-8.

[23] Fahey JW, Stephenson KK. Pinostrobin from honey and Thai ginger (Boesenbergia pandurata): A potent flavonoid inducer of mammalian phase 2 chemoprotective and antioxidant enzymes. $\mathrm{J}$ Agric Food Chem 2002; 50: 7472-6.

[24] Blasa M, Candiracci M, Accorsi A, Piacentini M, Albertini M, Piatti E. Raw Millefiori honey is packed full of antioxidants. Food Chem 2006; 97: 217-22.

[25] Nagai T, Inoue R, Kanamori N, Suzuki N, Nagashima T. Characterization of honey from different floral sources. Its functional properties and effects of honey species on storage of meat. Food Chem 2006; 97: 256-62.

[26] Perez RA, Iglesias MT, Pueyo E, Gonzalez M, de Lorenzo C. Amino acid composition and antioxidant capacity of Spanish honeys. J Agric Food Chem 2007; 55: 360-5.

[27] Gheldof N, Wang XH, Engeseth NJ. Buckwheat honey increases serum antioxidant capacity in humans. J Agric Food Chem 2003; 51: $1500-5$.

[28] Schramm DD, Karim M, Schrader HR, Holt RR, Cardetti M, Keen CL. Honey with high levels of antioxidants can provide protection to healthy human subjects. J Agric Food Chem 2003; 51: 17325 .

[29] Al-Waili NS. Effects of daily consumption of honey solution on hematological indices and blood levels of minerals and enzymes in normal individuals. J Med Food 2003; 6: 135-40.

[30] Baltrusaityte V, Venskutonis PR, Ceksteryte V. Radical scavenging activity of different floral origin honey and beebread phenolic extracts. Food Chem 2007; 101: 502-14.

[31] Kücük M, Kolayli S, Karaoglu S, Ulusoy E, Baltaci C, Candan F. Biological activities and chemical composition of three honeys of different types from Anatolia. Food Chem 2007; 100: 52634. 
[32] Vela L, de Lorenzo C, Pérez RA. Antioxidant capacity of Spanish honeys and its correlation with polyphenol content and other physicochemical properties. J Sci Food Agric 2007; 87: 1069-75.

[33] Gheldof N, Engeseth NJ. Antioxidant capacity of honeys from various floral sources based on the determination of oxygen radical absorbance capacity and inhibition of in vitro lipoprotein oxidation in human serum samples. J Agric Food Chem 2002; 50: 3050-5.

[34] Schramm D, Karim M, Schrader HR, Holt RR, Cardetti M, Keen CL. Honey with High Levels of Antioxidants Can Provide Protection to Healthy Human Subjects. J Agric Food Chem 2003; 51: 1732-5.

[35] Beretta G, Orioli M, Facino RM. Antioxidant and radical scavenging activity of honey in endothelial cell cultures (EA.hy926). Planta Med 2007; 73(11): 1182-9.

[36] Ahn MR, Kunimasa K, Kumazawa S, et al. Correlation between antiangiogenic activity and antioxidant activity of various components from propolis. Mol Nutr Food Res 2009; 53(5): 643-51.

[37] Natarajan K, Singh S, Burke Jr. TR, Grunberger D, Aggarwal BB. Caffeic acid phenethyl ester is a potent and specific inhibitor of activation of nuclear transcription factor NF- $\kappa$ B. Proc Natl Acad Sci USA 1996; 93(17): 9090-5.

[38] Staniforth V, Chiu LT, Yang NS. Caffeic acid suppresses UVB radiation-induced expression of interleukin-10 and activation of mitogen-activated protein kinases in mouse. Carcinogenesis 2006; 27(9): 1803-11.

[39] Weng MS, Ho YS, Lin JK. Chrysin induces G1 phase cell cycle arrest in C6 glioma cells through inducing p21Waf1/Cip1 expression: involvement of $\mathrm{p} 38$ mitogenactivated protein kinase. Biochem Pharmacol 2005; 69(12): 1815-27.

[40] Woo KJ, Jeong YJ, Park JW, Kwon TK. Chrysininduced apoptosis is mediated through caspase activation and Akt inactivation in U937 leukemia cells. Biochem Biophys Res Commun 2004; 325(4): 1215-22.

[41] Bestwick CS, Milne L. Influence of galangin on HL-60 cell proliferation and survival. Cancer Lett 2006; 243(1): 80-9.

[42] Kang T, Liang M. Studies on the inhibitory effects of quercetin on the growth of HL460 leukemia cells. Biochem Pharmacol 1997; 54: 1013-8.

[43] Robaszkiewicz A, Balcerczyk A, Bartosz G. Antioxidative and prooxidative effects of quercetin on A549 cells. Cell Biol Int 2007; 31(10): 1245-50.

[44] Braganhol E, Zamin LL, Canedo D. Antiproliferative effect of quercetin in the human U138MG glioma cell line. Anti Cancer Drugs 2006; 17(6): 663-71.

[45] Indap MA, Radhika S, Motiwale L, Rao KVK. Quercetin: antitumor activity and pharmacological manipulations for increased therapeutic gains. Indian J Pharm Sci 2006; 68(4): 465-9.

[46] Choi EJ, Bae SM, Ahn WS. Antiproliferative effects of quercetin through cell cycle arrest and apoptosis in human breast cancer MDA-MB-453 cells. Arch Pharm Res 2008; 31(10): 1281-5.

[47] Hsu YL, Kuo PL, Liu CF, Lin CC. Acacetin induced cell cycle arrest and apoptosis in human non-small cell lung cancer A549 cells. Cancer Lett 2004; 212(1): 53-60.

[48] Hsu YL, Kuo PL, Lin CC. Acacetin inhibits the proliferation of Hep G2 by blocking cell cycle progression and inducing apoptosis. Biochem Pharmacol 2004; 67(5): 823-9.

[49] Leung HWC, Lin CJ, Hour MJ, Yang WH, Wang MY, Lee HZ. Kaempferol induces apoptosis in human lung non-small carcinoma cells accompanied by an induction of antioxidant enzymes. Food Chem Toxicol 2007; 45(10): 2005-13.

[50] Bestwick CS, Milne L, Duthie SJ. Kaempferol induced inhibition of HL-60 cell growth results from a heterogeneous response, dominated by cell cycle alterations. Chem Biol Interact 2007; 170(2): 76-85.

[51] Kumar MAS, Nair M, Hema PS, Mohan J, Santhoshkumar TR. Pinocembrin triggers Bax-dependent mitochondrial apoptosis in colon cancer cells. Mol Carcinog 2007; 46(3): 231-41.

[52] Wang W, Heideman L, Chung CS, Pelling JC, Koehler KJ, Birt DF. Cell-cycle arrest at G2/M and growth inhibition by apigenin in human colon carcinoma cell lines. Mol Carcinog 2000; 28(2): 10210.

[53] Way TD, Kao MC, Lin JK. Apigenin induces apoptosis though proteasomal degradation of HER2/neu in HER2/neuoverexpressing breast cancer cells via the phosphatidylinositol-3kinase/Akt-dependent pathway. J Biol Chem 2004; 279: 447989.
[54] Zheng PW, Chiang LC, Lin CC. Apigenin induced apoptosis through p53-dependent pathway in human cervical carcinoma cells. Life Sci 2005; 76(12): 1367-79.

[55] Torkin R, Lavoie JF, Kaplan DR, Yeger H. Induction of caspasedependent, p53-mediated apoptosis by apigenin in human neuroblastoma. Mol Cancer Ther 2005; 4(1): 1-11.

[56] Chiang LC, Ng LT, Lin IC, Kuo PL, Lin CC. Antiproliferative effect of apigenin and its apoptotic induction in human Hep G2 cells. Cancer Lett 2006; 237(2): 207-14.

[57] Rakha MK, Nabil ZI, Hussein AA. Cardioactive and vasoactive effects of natural wild honey against cardiac malperformance induced by hyperadrenergic activity. J Med Food 2008; 11(1): 918.

[58] Nagyova A, Kudlackova MK, Horska A, et al. Lipid peroxidation in men after dietary supplementation with a mixture of antioxidant nutrients. Bratisl Lek Listy 2004; 105(7-8): 277-80.

[59] Yochum L, Kushi LH, Meyer K, Folsom AR. Dietary flavonoid intake and risk of cardiovascular disease in postmenopausal women. Am J Epidemiol 1999; 149: 943-9.

[60] Xia Z, Gu J, Ansley DM, Xia F, Yu J. Antioxidant therapy with Salvia miltiorrhiza decreases plasma endothelin-1 and thromboxane B2 after cardiopulmonary bypass in patients with congenital heart disease. J Thorac Cardiovasc Surg 2003; 126(5): 1404-10.

[61] Yoshizumi M, Tsuchiya K, Kirima K, Kyaw M, Suzaki Y, Tamaki T. Quercetin inhibits Shc- and phosphatidylinositol 3-kinasemediated c-Jun N-terminal kinase activation by angiotensin II in cultured rat aortic smooth muscle cells. Mol Pharmacol 2001; 60(4): 656-65.

[62] Sánchez M, Galisteo M, Vera R, et al. Quercetin downregulates NADPH oxidase, increases eNOS activity and prevents endothelial dysfunction in spontaneously hypertensive rats. J Hypertens 2006; 24(1): 75-84.

[63] Carlstrom J, Symons JD, Wu TC, Bruno RS, Litwin SE, Jalili T. A quercetin supplemented diet does not prevent cardiovascular complications in spontaneously hypertensive rats. J Nutr 2007; 137: 628-33.

[64] Duarte J, Palencia RP, Vargas F, et al. Antihypertensive effect of the avonoid quercetin in spontaneously hypertensive rats. $\mathrm{Br} \mathrm{J}$ Pharmacol 2001; 133: 117-24.

[65] Randi L, Edwards, Lyon T, et al. Quercetin Reduces Blood Pressure in Hypertensive Subjects. J Nutr 2007; 137: 2405-11.

[66] Li GR, Wang HB, Qin GW, et al. Acacetin, a natural flavone, selectively inhibits human atrial repolarization potassium currents and prevents atrial fibrillation in dogs. Circulation 2008; 117: 2449-57.

[67] Iraz M, Fadillioglu E, Tasdemir S, Erdogan S. Role of vagal activity on bradicardic and hypotensive effects of caffeic acid phenethyl ester (CAPE). Cardiovasc Toxicol 2005; 5(4): 391-6.

[68] Xu YC, Yeung DKY, Man RYK, Leung SWS. Kaempferol enhances endothelium-independent and dependent relaxation in the porcine coronary artery. Mol Cell Biochem 2006; 287: 61-7.

[69] Kim DS, Ha KCK Won DY, et al. Kaempferol protects ische$\mathrm{mia} /$ reperfusion-induced cardiac damage through the regulation of endoplasmic reticulum stress. Immunopharmacol Immunotoxicol 2008; 30(2): 257-70.

[70] Lysias-Derrida C. Galangal: Lesser galangal [on-line]. 2006. Available: http://www.mdidea.com/products/new/new003.html. [cited 2009 sep 15].

[71] Ha SK, Lee P, Park JA, et al. Apigenin inhibits the production of NO and PGE2 in microglia and inhibits neuronal cell death in a middle cerebral artery occlusion-induced focal ischemia mice model. Neurochem Int 2008; 52: 878-86.

[72] Losi G, Puia G, Garzon G, Vuono MC, Baraldi M. Apigenin modulates GABAergic and glutamatergic transmission in cultured cortical neurons. Eur J Pharmacol 2004; 502: 41-6.

[73] Liu R, Gao M, Zhi-Hong Y, Guan-Hua D. Pinocembrin protects rat brain against oxidation and apoptosis induced by ischemiareperfusion both in vivo and in vitro. Eur J Pharmacol 2008; 1216: 104-15.

[74] Hong-Mei Guang, Guan-Hua Du. Protections of pinocembrin on brain mitochondria contribute to cognitive improvement in chronic cerebral hypoperfused rats. Eur J Pharmacol 2006; 542: 77-83.

[75] Estevinho L, Pereira AP, Moreira L, Dias LG, Pereira E. Antioxidant and antimicrobial effects of phenolic compounds extracts of Northeast Portugal honey. Food Chem Toxicol 2008; 46(12): 37749. 
[76] Wahdan HA. Causes of the antimicrobial activity of honey. J Infect 1998; 26(1): 26-31.

[77] Taormina PJ, Niemira BA, Benchat LR. Inhibitory activity of honey against food borne pathogens as influenced by the presence of hydrogen peroxide and level of antioxidant power. Int J Food Microbiol 2001; 69: 217-55.

[78] Jackson AS, Cooper R, Burton N. Free radical production and quenching in honeys with wound healing potential. J Antimicrob Chemother 2006; 58(4): 773-7.
[79] Rasmussen CN, Wang XH, Leung S, Andrae-Nightingale LM, Schmidt SJ, Engeseth NJ. Selection and use of honey as an antioxidant in a French salad dressing system. J Agric Food Chem 2008; 56(18): 8650-7.

[80] Mckibben J, Engeseth NJ. Honey as a Protective Agent against Lipid Oxidation in Ground Turkey. J Agric Food Chem 2002; 50: 592-5.

Received: October 26, 2009

(C) Khalil et al.; Licensee Bentham Open.

This is an open access article licensed under the terms of the Creative Commons Attribution Non-Commercial License (http://creativecommons.org/licenses/ by-nc/3.0/) which permits unrestricted, non-commercial use, distribution and reproduction in any medium, provided the work is properly cited. 